\title{
Graphene-Like Bilayer Hexagonal Silicon Polymorph
}

\author{
Jaeil Bai ${ }^{1}$, Hideki Tanaka ${ }^{2}$, and Xiao Cheng Zeng ${ }^{1}(\bowtie)$ \\ ${ }^{1}$ Department of Chemistry and Nebraska Center for Materials and Nanoscience, University of Nebraska-Lincoln, Lincoln, Nebraska \\ 68588, USA \\ ${ }^{2}$ Department of Chemistry, Okayama University, Okayama 700-8530, Japan \\ Received: 18 June 2010 / Revised: 3 August 2010 / Accepted: 9 August 2010 \\ (C) The Author(s) 2010. This article is published with open access at Springerlink.com
}

\begin{abstract}
We present molecular dynamics simulation evidence for a freezing transition from liquid silicon to quasi-twodimensional (quasi-2D) bilayer silicon in a slit nanopore. This new quasi-2D polymorph of silicon exhibits a bilayer hexagonal structure in which the covalent coordination number of every silicon atom is four. Quantum molecular dynamics simulations show that the stand-alone bilayer silicon (without the confinement) is still stable at $400 \mathrm{~K}$. Electronic band-structure calculations suggest that the bilayer hexagonal silicon is a quasi-2D semimetal, similar to a graphene monolayer, but with an indirect zero band gap.
\end{abstract}

\section{KEYWORDS}

Bilayer hexagonal silicon, slit pore, semimetal, two-dimensional polymorph

Low-dimensional silicon nanostructures are known to possess properties that are markedly different from the bulk. Silicon nanowires, for example, can exhibit notable quantum-size effects if the carriers are confined to dimensions less than the de Broglie wavelength [1]. To exploit this novel quantum-size effect, considerable attention has been devoted to developing and fabricating low-dimensional silicon nanostructures.

Bulk silicon possesses a cubic diamond structure in which every Si atom has four nearest neighbors. In the solid state (ambient conditions) silicon is a semiconductor, whereas in the liquid state silicon is a metal with a typical coordination number of $6-7[2,3]$. It is known that silicon and ice $I_{\mathrm{h}}$ share some similarities in structural transitions [4]. For example, both silicon and ice $I_{\mathrm{h}}$ belong to the "tetrahedral network" family as both have the cubic diamond structure in the solid state. Upon melting, both silicon and ice $I_{\mathrm{h}}$ shrink in volume. More interestingly, when confined to a quasione-dimensional (quasi-1D) nanopore (e.g., a carbon nanotube) both liquid silicon and water can freeze into single-walled polygonal nanotubes below their melting points $[5,6]$. The existence of single-walled polygonal ice nanotubes has been recently confirmed experimentally [7-10] and single-walled hexagonal silicon nanotubes containing a metal dopant have also been fabricated in the laboratory [11].

This prompts a question: if the liquid silicon is confined to a slit nanopore can new polymorphs of quasi-2D silicon form on freezing, as is observed for liquid water [12-14]? In this article, we report molecular dynamics simulation evidence for the formation of a bilayer hexagonal silicon polymorph on freezing liquid

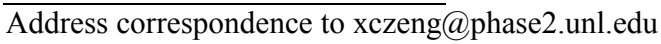


silicon in a slit nanopore. Moreover, we performed a quantum molecular dynamics simulation of standalone bilayer silicon at $400 \mathrm{~K}$ to confirm its structural stability. Electronic and phonon properties of the new quasi-2D polymorph of silicon were computed based on density functional theory (DFT) calculations [15-17].

In a previous study, we performed a classical molecular dynamics (MD) simulation of the freezing of liquid silicon in a nanoscale slit [18]. The simulation system consists of a slit nanopore with two planar walls which were assumed to be smooth and rigid. The wall-wall separation was set to accommodate just two layers of silicon. The Stillinger-Weber potential model [19] was used for the silicon and the 9-3 Lennard-Jones (LJ) potential for the silicon-wall interaction. Through the MD simulation of the freezing transition, we observed that the lower temperature solid phase was indeed a bilayer hexagonal silicon polymorph (Fig. 1). To show that the formation of the bilayer silicon is independent of the models employed for the silicon and slit pore, we performed two new MD simulations. First, we used the Tersoff potential model of silicon [20] and the 9-3 LJ potential for the nanopore. The constant lateral-pressure and temperature ensemble was adopted [12-15]. The lateral area of the simulation cell was allowed to change and the lateral pressure was set to be $50 \mathrm{MPa}$. The periodic boundary conditions were applied only in the lateral directions ( $x$ and $y$ ) parallel to the two walls. The width of the slit nanopore was fixed at $8.08 \AA$. The simulation cell contained

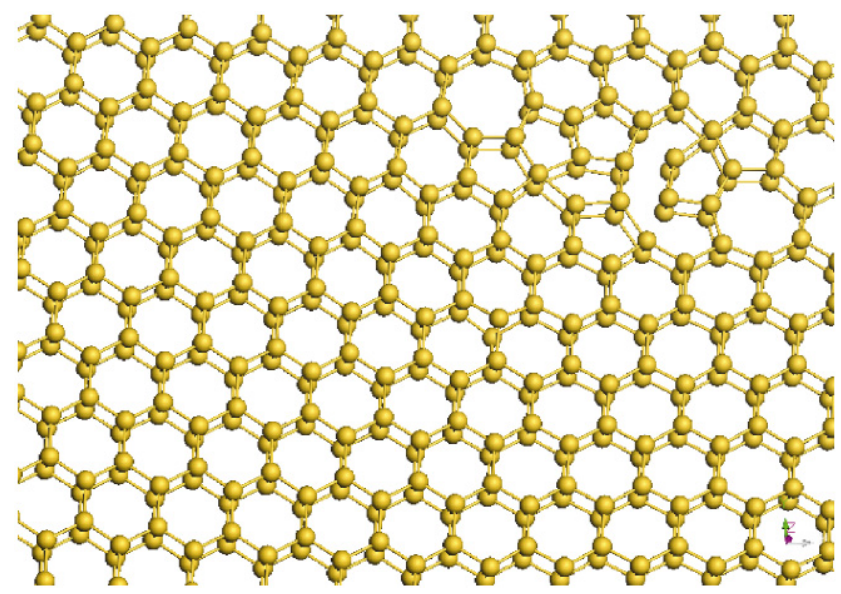

Figure 1 A quasi-2D bilayer hexagonal silicon polymorph formed spontaneously in a nanoscale slit at $1659 \mathrm{~K}$. The StillingerWeber potential was employed for silicon
$512 \mathrm{Si}$ atoms. Initially, the liquid silicon was equilibrated at $3018 \mathrm{~K}$, followed by an instantaneous temperature decrease to $2137 \mathrm{~K}$. A bilayer hexagonal silicon polymorph was observed after a few million MD steps (each MD time step was $0.3 \mathrm{fs}$ ). This quasi-2D hexagonal structure typically contains a few topologic defects such as pentagons and heptagons, a dominant structural feature in bilayer amorphous ice [13, 14]. Next, we performed a MD simulation using a structured wall, that is, a monolayer carbon graphene. A total of $2 \times$ $384=768 \mathrm{C}$ atoms and $308 \mathrm{Si}$ atoms were included in the simulation cell. The Tersoff $\mathrm{C} / \mathrm{Si}$ potential parameters [15] were used to describe the C/Si interaction. The lateral dimensions of the simulation cell were fixed at $34.95 \AA \times 29.87 \AA$. The width of the nanopore was fixed at $7 \AA$. Liquid silicon was initially equilibrated at $3018 \mathrm{~K}$ and then the temperature was instantaneously reduced to $1886 \mathrm{~K}$. Again, the bilayer silicon formed spontaneously between the two structured walls. A snapshot of the bilayer hexagonal silicon is shown in Figs. 2(a) and 2(b) (top and side view, respectively), and snapshots of liquid silicon are shown in Figs. 2(c) and $2(\mathrm{~d})$.

To further confirm the stability of the bilayer hexagonal silicon polymorph, we performed a quantum MD simulation for a stand-alone bilayer silicon (without the confinement) using the CASTEP code [21]. The simulation cell contained $64 \mathrm{Si}$ atoms. Temperature and pressure were constrained to be $400 \mathrm{~K}$ and zero, respectively, using the Nosé-Andersen method. For the quantum MD simulation, a DFT method within the generalized-gradient approximation with the PerdewBurke-Ernzerhof (PBE) functional [22] was used together with the ultrasoft pseudopotential [23]. The kinetic energy cutoff was set to be $120 \mathrm{eV}$. The MD time step was $0.8 \mathrm{fs}$. A snapshot of the quantum MD simulation at $10 \mathrm{ps}$ is shown in Fig. 3. No apparent structural distortion from the bilayer hexagonal structure was observed except for slight vibrational displacements of the silicon atoms from their ideal lattice positions. Although the bilayer hexagonal silicon is not the most stable thermodynamic phase at zero pressure and $400 \mathrm{~K}$ (the 3D cubic diamond phase is), this quantum MD simulation suggests that the bilayer silicon is still stable in a vacuum. Apparently, an appreciable free-energy barrier separates the bilayer

\section{Springer}




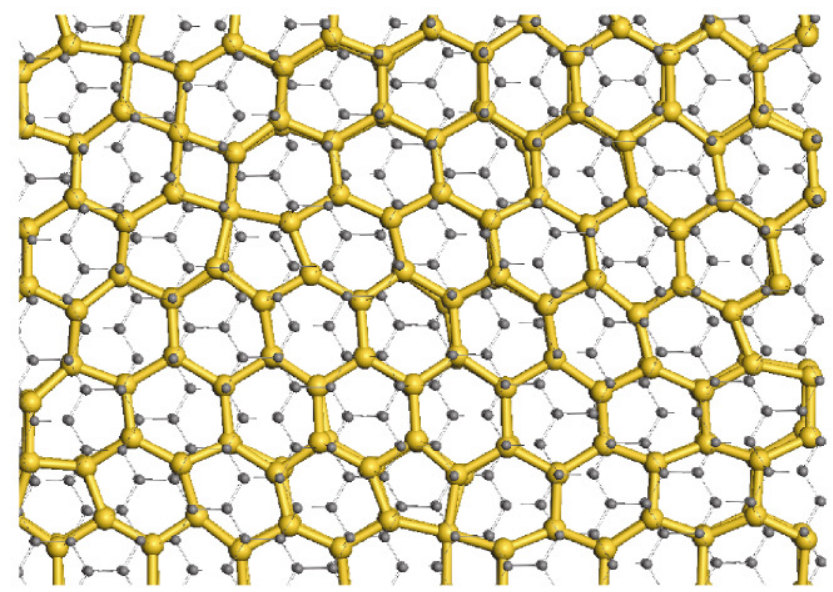

(a)

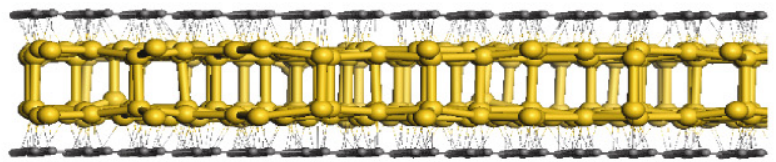

(b)

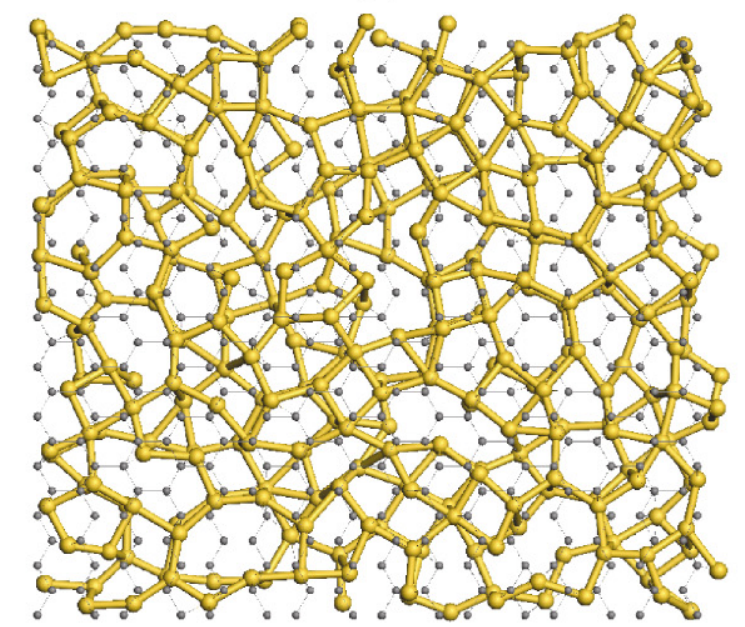

(c)

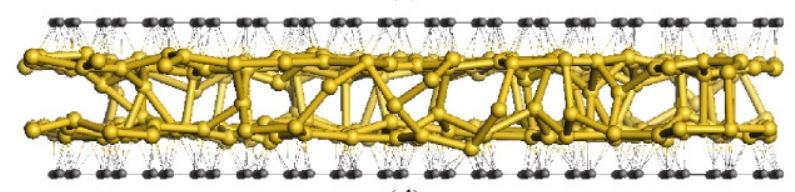

(d)

Figure 2 Top (a) and side (b) views of the bilayer hexagonal silicon, and top (c) and side (d) views of liquid silicon. The two walls are carbon graphene monolayers (gray color)

phase from the most stable 3D diamond phase so that the bilayer phase is metastable at room temperature.

Another piece of supporting evidence for the stability of the bilayer hexagonal structure in a vacuum was provided by DFT calculations of the harmonic vibrational frequencies of a series of finite-size bilayer hexagonal clusters with increasing size (see Fig. 4). To

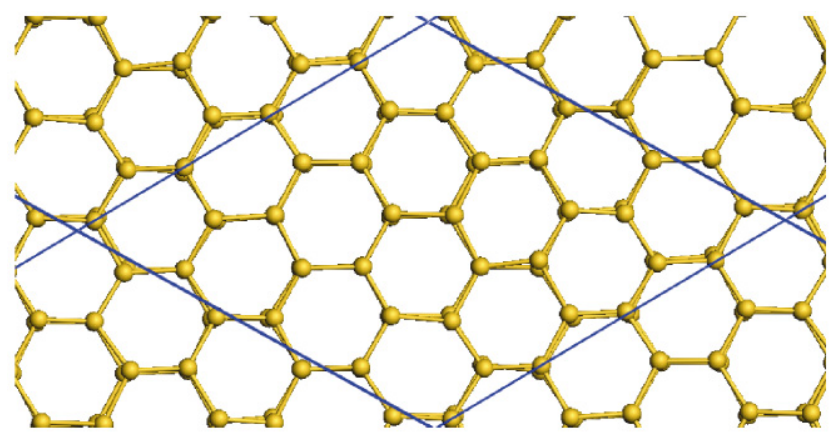

(a)

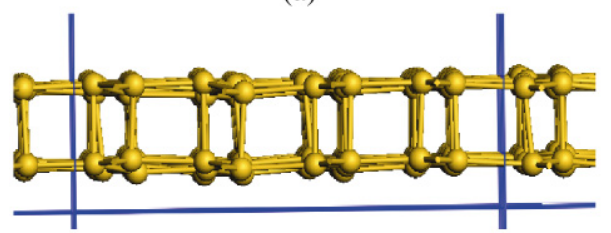

(b)

Figure 3 Top (a) and side (b) views of a snapshot of quantum MD simulations of bilayer hexagonal silicon at $10 \mathrm{ps}$. Blue lines denote the supercell. The supercell parameters are $a=b=16.60 \AA$, $c=14.7 \AA, \alpha=\beta=90^{\circ}$, and $\gamma=120^{\circ}$

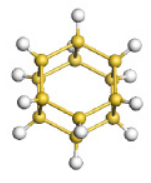

(a)

$$
\text { a }
$$

(d)

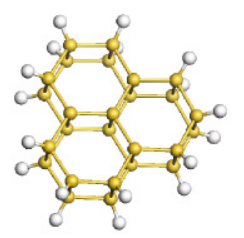

(b)

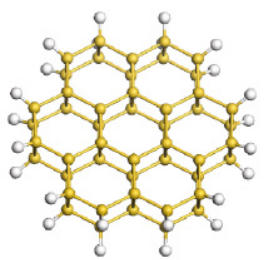

(c)
Figure 4 Bilayer hexagonal clusters: (a) $\mathrm{Si}_{12} \mathrm{H}_{12}$, (b) $\mathrm{Si}_{26} \mathrm{H}_{18}$, (c) $\mathrm{Si}_{48} \mathrm{H}_{24}$, (d) $\mathrm{Si}_{74} \mathrm{H}_{30}$, and (e) $\mathrm{Si}_{84} \mathrm{H}_{32}$. Si atoms at the perimeter are passivated by hydrogen atoms. (f) An optimized structure of the core in (e) without hydrogen passivation. Green colored atoms in (f) indicate that the interior hexagonal bilayer cluster maintains the same geometric features as the 2D bilayer polymorph, even with the reconstruction occuring at the edge

remove the "surface" reconstruction effect, all silicon atoms at the perimeter were passivated by hydrogen atoms. The bare silicon clusters themselves may be viewed as being cut out of the bilayer silicon by a perpendicular circular cut. As such, the coordination number of every $\mathrm{Si}$ atom is four-fold except for those 
at the perimeter. The geometry of the clusters was first optimized using an all-electron DFT method with the gradient-correct Becke-Lee-Yang-Parr (BLYP) exchange correlation functional [24-26] and the double numerical basis sets and polarization function (DNP), implemented in the $\mathrm{DMol}^{3}$ software package [27-28]. Subsequent frequency calculations showed that all the finite-size hexagonal bilayer silicon clusters are local minima with no imaginary frequencies. Without the hydrogen passivation, the interior part of the hexagonal-bilayer clusters maintained the same geometric features as the 2D bilayer polymorph (see the green colored atoms in Fig. 4(b)), even though reconstruction occured at the edge of the cluster. Table 1 shows the calculated HOMO-LUMO gaps of hexagonal bilayer silicon clusters with hydrogen passivation. It can be seen that as the size of cluster increases the HOMO-LUMO gap of the cluster decreases, consistent with the semimetallic behavior of the cluster when its size goes to infinity.

Having demonstrated the stability of the bilayer hexagonal silicon polymorph, it is interesting to explore the electronic and phonon properties of this new quasi-2D polymorph of silicon. Note that the hexagonal structure of the bilayer silicon is very similar to that of a carbon graphene monolayer. The electronic properties of graphene monolayers have received considerable attention $[29,30]$ due to the close resemblance of their band spectrum to the Dirac spectrum for massless fermions [31-32]. The graphene form of carbon is a semimetal with (direct) zero band gap. Hence, it is interesting to investigate graphene-like silicon structures [33-36]. To this end, we computed the band structures for both perfect bilayer hexagonal silicon and an artificial monolayer hexagonal silicon (silicon graphene), as shown in Figs. 5(a) and 5(b). The band structures were computed based on the DFT method with the PBE functional and the norm-conserving pseudopotential. The kinetic energy cutoff was set to be $330 \mathrm{eV}$. The Brillouin zone was sampled with $(12 \times$ $12 \times 1) k$ points of a Monkhorst-Pack grid [37]. For the bilayer silicon, the geometry was first relaxed until the stress tolerance criteria of $0.5 \times 10^{-6} \mathrm{eV} /$ atom total energy and $0.001 \mathrm{GPa}$ were met. The optimized supercell parameters were $a=b=4.075 \AA, c=20 \AA$, $\alpha=\beta=90^{\circ}$, and $\gamma=120^{\circ}$. For the artificial monolayer
Table 1 Computed HOMO-LUMO gaps of the hexagonal bilayer clusters shown in Figs. 4(a)-4(e)

\begin{tabular}{cc}
\hline Clusters & HOMO-LUMO gap (eV) \\
\hline (a) $\mathrm{Si}_{12} \mathrm{H}_{12}$ & 2.311 \\
(b) $\mathrm{Si}_{26} \mathrm{H}_{18}$ & 1.981 \\
(c) $\mathrm{Si}_{48} \mathrm{H}_{24}$ & 1.107 \\
(d) $\mathrm{Si}_{74} \mathrm{H}_{30}$ & 1.02 \\
(e) $\mathrm{Si}_{84} \mathrm{H}_{32}$ & 0.91 \\
\hline
\end{tabular}

hexagonal silicon, geometry relaxation was not undertaken because the structure itself is unstable in a vacuum. The band structures of the monolayer hexagonal silicon were computed based on the same supercell parameters $a, b$, and $c$ for the bilayer silicon. In addition, we computed the band structures of an ideally cleaved $\operatorname{Si}(1 \times 1 \times 1)$ bilayer (Fig. 5(c)). The unit cell of the $\mathrm{Si}(1 \times 1 \times 1)$ bilayer has four $\mathrm{Si}$ atoms as shown in Fig. $5(\mathrm{~d})$. Note that the $\mathrm{Si}(1 \times 1 \times 1)$ bilayer also exhibits a hexagonal structure but the atomic layer itself is not flat.

For the monolayer hexagonal silicon, Fig. 5(b) shows that the conduction band minimum (CBM) just touches the valence band maximum (VBM) at the zone edge $\mathrm{K}$ point and the band crossing occurs exactly at the Fermi level. This band structure looks exactly the same as that of the carbon graphene near the zone

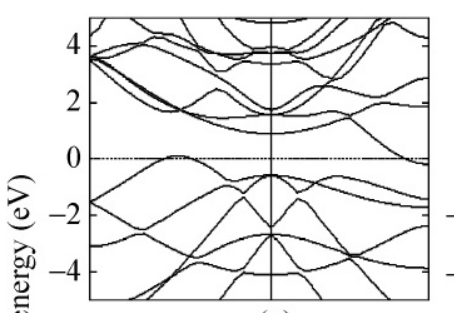

(a)

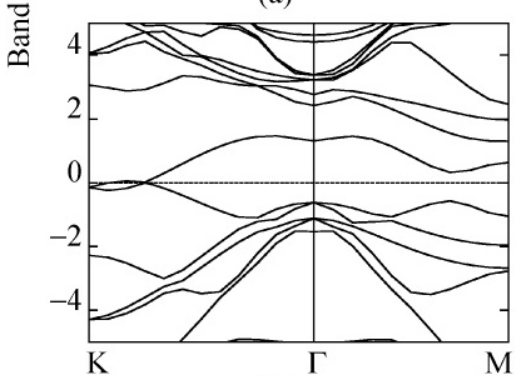

(c)

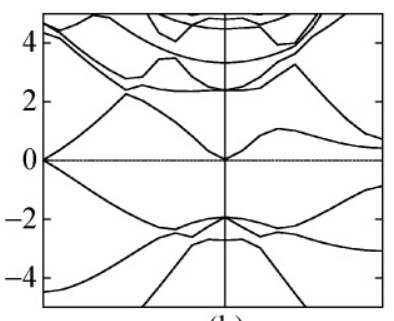

(b)

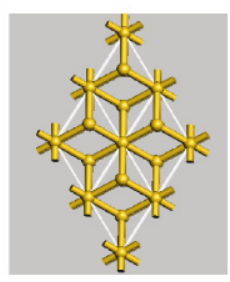

(d)
Figure 5 The computed electronic band structures of (a) the bilayer hexagonal silicon, (b) the (unstable) monolayer hexagonal silicon, and (c) an ideally cleaved Si $(1 \times 1 \times 1)$ bilayer whose structure is shown in (d) 
edge K point [30]. Thus, like the carbon graphene the (unstable) monolayer hexagonal silicon is also a semimetal with (direct) zero band gap. For the $\mathrm{Si}(1 \times 1 \times 1)$ bilayer, the band crossing also occurs at the Fermi level, but slightly shifts off the zone edge $\mathrm{K}$ point. Nevertheless, the band structure also suggests that the $\operatorname{Si}(1 \times 1 \times 1)$ bilayer is a semimetal with (direct) zero band gap. In stark contrast, the band structure of the bilayer hexagonal silicon exhibits an indirect overlap between the CBM and VBM, as shown in Fig. 5(a). This suggests that the bilayer hexagonal silicon is a semimetal but with (indirect) zero band gap. To the best of our knowledge, a quasi-2D semimetal with indirect zero band gap has not previously been reported in the literature.

Additional insight into the electronic properties of the bilayer hexagonal silicon may be gained from an analysis of its structure. First, the local geometry of the bilayer silicon is quite distorted from the local tetrahedral network of the bulk counterpart. Within one layer, the Si-Si bond length is $2.39 \AA$ (after DFT optimization) whereas between two layers the $\mathrm{Si}-\mathrm{Si}$ bond length is slightly larger $(2.42 \AA)$. Both bond lengths are longer than the Si-Si bond length $(2.34 \AA)$ in the bulk, due largely to the distorted electron distribution in the bilayer silicon necessary to maintain the planar structure. In addition, Mulliken population analysis further confirms that the electron densities of the intralayer Si-Si bonds are significantly higher than those of the interlayer $\mathrm{Si}-\mathrm{Si}$ bonds. It is therefore likely that the interlayer $\mathrm{Si}-\mathrm{Si}$ bonds are weaker than the intralayer $\mathrm{Si}-\mathrm{Si}$ bonds.

The potential energy of the bilayer hexagonal silicon is $0.044 \mathrm{eV} /$ atom lower than that of the bilayer $\mathrm{Si}$ $(1 \times 1 \times 1)$ structure but $0.91 \mathrm{eV} /$ atom higher than that of bulk silicon. This shows that the bilayer hexagonal silicon with its distorted tetrahedral bonding is energetically more favorable than the bilayer $\mathrm{Si}(1 \times 1 \times 1)$ structure with no distorted tetrahedral bonding.

Lastly, the phonon dispersion curves (Fig. 6(a)) of the bilayer hexagonal silicon were computed using the finite displacement method [38] as well as the DFT method for the band-structure calculations. Here, the supercell contained $38 \mathrm{Si}$ atoms. For the purpose of comparison, we also used the same method to compute the phonon dispersion curves of bulk silicon.
The standard six branch phonon dispersion curves were reproduced (Fig. 6(b)) in order to validate the theoretical method. For the bilayer hexagonal silicon, twelve phonon dispersion curves can be found, twice as many as those for the bulk counterpart. Moreover, at the $\Gamma$ point, four high frequency modes are found for the bilayer silicon whereas only one is found for bulk silicon. Interestingly, the three phonon dispersion curves near the $\Gamma$ point are very similar to those of the planar monolayer silicon reported by Ciraci and coworkers [34]. The fact that no imaginary frequencies were observed in the phonon modes confirms that the bilayer hexagonal silicon is a stable quasi-2D polymorph.

In conclusion, we have performed MD simulations of liquid silicon confined to slit nanopores and demonstrated the formation of a new quasi-2D silicon

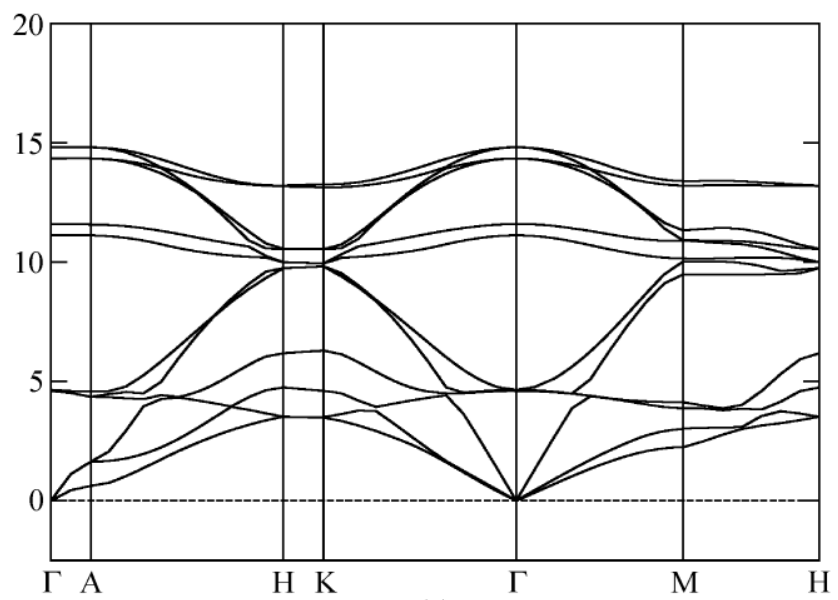

(a)

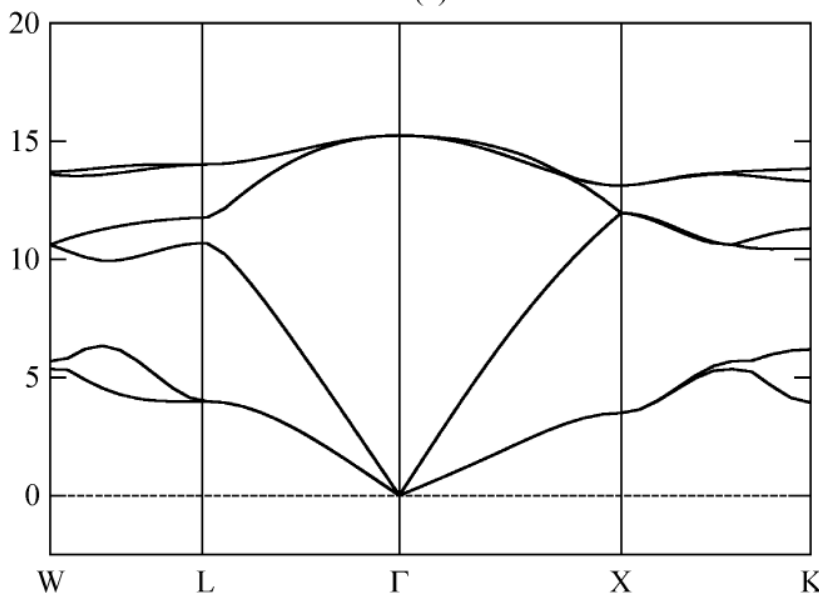

(b)

Figure 6 Phonon dispersion curves along high symmetry directions for (a) the bilayer hexagonal silicon and (b) bulk silicon 
polymorph at low temperature. This freezing transition is independent of the silicon potential model selected and the model for the nanopores. Quantum MD simulations of a stand-alone bilayer hexagonal silicon at finite temperature further confirm the stability of this new silicon polymorph. Electronic band-structure calculations suggest that the bilayer hexagonal silicon is a semimetal with zero band gap. Whether or not the bilayer hexagonal silicon can actually be synthesized is certainly an open question. In our MD simulations, the model carbon walls are inert. In reality, however, liquid silicon would react with graphite or carbon fibers to form $\operatorname{SiC}[39,40]$. Hence, it is important to search for a refractory material with a melting point much higher than silicon and yet being inert to molten silicon. Finally, we remark that our simulations provide additional evidence that silicon and ice belong to a generic "tetrahedral network" family, as both exhibit nearly the same isomorphic structures. We note also that the free-standing bilayer hexagonal ice has been recently observed in the laboratory [41].

\section{Acknowledgements}

This work was supported by grants from the Department of Energy (DOE) (No. DE-FG02-04ER46164), the Nebraska Research Initiative, and the University of Nebraska's Holland Computing Center.

Open Access: This article is distributed under the terms of the Creative Commons Attribution Noncommercial License which permits any noncommercial use, distribution, and reproduction in any medium, provided the original author(s) and source are credited.

\section{References}

[1] Harrison, P. Quantum Wells, Wires and Dots: Theoretical and Computational Physics; Wiley: New York, 2000.

[2] Jank, W.; Hafner, J. Structural and electronic properties of the liquid polyvalent elements: The group-IV elements $\mathrm{Si}$, Ge, Sn, and Pb. Phys. Rev. B 1990, 41, 1497-1515.

[3] Jakse, N.; Henne, L.; Price, D. L.; Krishnan, S.; Key, T.; Artacho, E.; Glorieux, B.; Pasturel, A.; Saboungi, M. L. Structural changes on supercooling liquid silicon. Appl.
Phys. Lett. 2003, 83, 4734-4736.

[4] Sastry, S.; Angell, C. A.; Liquid-liquid phase transition in supercooled silicon. Nat. Mater. 2003, 2, 739-743.

[5] Bai, J.; Zeng, X. C.; Tanaka, H.; Zeng, J. Y. Metallic singlewalled silicon nanotubes. Proc. Natl. Acad. Sci. USA 2004, 101, 2664-2668.

[6] Koga, K.; Gao, G. T.; Tanaka, H.; Zeng, X. C. Formation of ordered ice nanotubes inside carbon nanotubes. Nature 2001, 412, 802-805.

[7] Ghosh, S.; Ramanathan, K. V.; Sood, A. K. Water at nanoscale confined in single-walled carbon nanotubes studied by NMR. Europhys. Lett. 2004, 65, 678-684.

[8] Kolesnikov, A. I.; Zanotti, J. M.; Loong, K.; Thyagarajan, P.; Morsvsky, A. P.; Loutfy, R. O.; Burnham, C. J. Anomalously soft dynamics of water in a nanotube: A revelation of nanoscale confinement. Phys. Rev. Lett. 2004, 93, 035503.

[9] Maniwa, Y.; Kataura, H.; Abe, M.; Udaka, A.; Suzuki, S.; Achiba, Y.; Kira, H.; Matsuda, K.; Kadowaki, H.; Okabe, Y. Ordered water inside carbon nanotubes: Formation of pentagonal to octagonal ice-nanotubes. Chem. Phys. Lett. 2005, 401, 534-538.

[10] Byl, O.; Liu, J. C.; Wang, Y.; Yim, W. L; Johnson, J. K.; Yates, J. T. Jr. Unusual hydrogen bonding in water-filled carbon nanotubes. J. Am. Chem. Soc. 2006, 128, 1209012097.

[11] Saranin, A. A.; Zotov, A. V.; Kotlyar, V. G.; Kasyanova, T. V.; Utas, O. A.; Okado, H.; Katayama, M.; Oura, K. Ordered arrays of Be-encapsulated Si nanotubes on $\mathrm{Si}(111)$ surface. Nano. Lett. 2004, 4, 1469-1473.

[12] Koga, K.; Zeng, X. C.; Tanaka, H. Freezing of confined water: A bilayer ice phase in hydrophobic nanopores. Phys. Rev. Lett. 1997, 79, 5262-5265.

[13] Koga, K.; Tanaka, H.; Zeng, X. C. First-order transition in confined water between high-density liquid and low-density amorphous phases. Nature 2000, 408, 564-567.

[14] Bai, J.; Zeng, X. C.; Koga, K.; Tanaka, H. Formation of quasitwo-dimensional bilayer ice in hydrophobic slit: A possible candidate for ice XIII? Mol. Simul. 2003, 29, 619-626.

[15] Payne, M. C.; Teter, M. P.; Allan, D. C.; Arias, T. A.; Joannopoulos, J. D. Iterative minimization techniques for $a b$ initio total-energy calculations: Molecular dynamics and conjugate gradients. Rev. Mod. Phys. 1992, 64, 1045-1097.

[16] Kresse, G.; Hafner, J. Ab initio molecular dynamics for liquid metals. Phys. Rev. B 1993, 47, 558-561.

[17] Kresse, G.; Furthmüller, J. Efficient iterative schemes for $a b$ initio total-energy calculations using a plane-wave basis set. Phys. Rev. B 1996, 54, 11169-11186.

[18] Bai, J. Novel Low Dimensional Silicon and Water Nanostructures. Ph.D. Dissertation, University of Nebraska-Lincoln, Lincoln, NE, USA, 2004. 
[19] Stillinger, F. H.; Weber, T. A. Computer simulation of local order in condensed phases of silicon. Phys. Rev. B 1985, 31, 5262-5271.

[20] Tersoff, J. Modeling solid-state chemistry: Interatomic potentials for multicomponent systems. Phys. Rev. B 1989, 39, 5566-5568.

[21] CASTEP is available from Accelrys Inc. http://accelrys.com/ products/materials-studio/quantum-and-catalysis-software.html

[22] Perdew, J. P.; Burke, K.; Ernzerhof, M. Generalized gradient approximation made simple. Phys. Rev. Lett. 1996, 77, 3865-3868.

[23] Vanderbilt, D. Soft self-consistent pseudopotentials in a generalized eigenvalue formalism. Phys. Rev. B 1990, 41, 7892-7895.

[24] Becke, A. D. Density-functional exchange-energy approximation with correct asymptotic behavior. Phys. Rev. A 1988, 38, 3098-3100.

[25] Perdew, J. P. Density-functional approximation for the correlation energy of the inhomogeneous electron gas. Phys. Rev. B 1986, 33, 8822-8824.

[26] Lee, C.; Yang, W.; Parr, R. G. Development of the ColleSalvetti correlation-energy formula into a functional of the electron density. Phys. Rev. B 1988, 37, 785-789.

[27] Delly, B. From molecules to solids with the $\mathrm{DMol}^{3}$ approach. J. Chem. Phys. 2000, 113, 7756.

[28] $\mathrm{DMol}^{3}$ is available from Accelrys Inc. http://accelrys.com/ products/materials-studio/quantum-and-catalysis-software.html

[29] Geim, A. K.; Novoselov, K. S. The rise of graphene. Nat. Mater. 2007, 6, 183-191.

[30] Katsnelson, M. L. Graphene: Carbon in two dimensions. Mater. Today 2007, 10, 20-27.

[31] Semenoff, G. W. Condensed-matter simulation of a threedimensional anomaly. Phys. Rev. Lett. 1984, 53, 2449-2452.
[32] Haldane, F. D. M. Model for a quantum Hall effect without Landau levels: Condensed-matter realization of the "parity anomaly". Phys. Rev. Lett. 1984, 61, 2015-2018.

[33] Nakano, H.; Mitsuoka, T.; Harada, M.; Horibuchi, K.; Nozaki, H.; Takahashi, N.; Nonaka, T.; Seno, Y.; Nakamura, H. Soft synthesis of single-crystal silicon monolayer sheets. Angew. Chem. Int. Ed. 2006, 45, 6303-6306.

[34] Cahangirov, S.; Topsakal, M.; Aktürk, E.; Şahin, H.; Ciraci, $\mathrm{S}$. Honeycomb structures of silicon and germanium. Phys. Rev. Lett. 2009, 102, 236804.

[35] Lebègue, S.; Eriksson, O. Nonadiabatic potential-energy surfaces by constrained density-functional theory. Phys. Rev. B 2009, 79, 115409.

[36] Wang, S. Studies of physical and chemical properties of twodimensional hexagonal crystals by first-principles calculation. J. Phys. Soc. Japan, 2010, 79, 064602.

[37] Monkhorst, H. J.; Pack, J. D. Special points for Brillouinzone integrations. Phys. Rev. B 1976, 13, 5188-5192.

[38] Ackland, G. J.; Warren, M. C.; Clark, S.J. Practical methods in ab initio lattice dynamics. J. Phys.: Condens. Matter. 1997, 9, 7861-7872.

[39] Schulte-Fischedick, J.; Zern, A.; Mayer, J.; Rühle, M.; Frieß, M.; Krenkel, W. The morphology of silicon carbide in $\mathrm{C} / \mathrm{C}-$ SiC composites. Mater. Sci. Eng. A 2002, 332, 146-152.

[40] Sangsuwan, P.; Tewari, S. N.; Gatica, J. E.; Singh, M.; Dickerson, R. Reactive infiltration of silicon melt through microporous amorphous carbon preforms. Metall. Mater. Trans. B 1999, 30, 933-944.

[41] Kimmel, G. A.; Matthiesen, J.; Baer, M.; Mundy, C. J.; Petrik, N. G.; Smith, R. S.; Dohnalek, Z.; Kay, B. D. No confinement needed: Observation of a metastable hydrophobic wetting two-layer ice on graphene. J. Am. Chem. Soc. 2009, $131,12838-12844$. 